\title{
Hydrogen Bonding and Symmetry Relationships in Quantum Materials
}

\author{
Efrain E. Rodriguez (efrain@umd.edu)
}

Chemistry and Biochemistry, University of Maryland, College Park, MD 20742

Margaret C. Etter's seminal work on hydrogen bonding has left a deep and broad impact on crystal chemistry and crystallography. The Etter Rules for describing patterns in hydrogen bonding have been invoked for a wide variety of chemical problems including supramolecular chemistry, materials chemistry, catalysis, and solid state chemistry. In honor of Margaret C. Etter, I will present some work on inorganic materials where hydrogen bonding plays a role in stabilizing structures that would otherwise not exist. The work of my research group has focused on the synthesis and structural studies of compounds broadly known as quantum materials. Although resistant to a precise definition, quantum materials broadly encompass categories such as superconductors, topological insulators, highly-correlated electron systems, and materials that express frustrated magnetism. In this lecture I will focus on the layered ironbased superconductors and the intercalated phases such as $\left(\mathrm{Li}_{1-x} \mathrm{Fe}_{x} \mathrm{OH}\right) \mathrm{FeCh}$, [ $\mathrm{Na}_{1-}$ $\left.{ }_{x} \mathrm{Fe}_{\mathrm{x}}(\mathrm{OH})_{2}\right] \mathrm{FeCh}$, and $\left[\mathrm{Li}\left(\mathrm{C}_{2} \mathrm{H}_{8} \mathrm{~N}_{2}\right)_{\mathrm{y}}\right] \mathrm{FeCh}$ where $\mathrm{Ch}$ is $\mathrm{S}$ and $\mathrm{Se}$. We propose that hydrogen bonding of the type $\mathrm{N}-\mathrm{H} \cdots \mathrm{Ch}$ and $\mathrm{O}-\mathrm{H} \cdots \mathrm{Ch}$ stabilize the growth of these layered iron chalcogenides. Due to the preparation from hydrothermal and solvothermal syntheses, the crystal growth of these layers involves several intermediate phases involving hydrogen bonding as evidenced by in situ X-ray diffraction studies. Finally, I will discuss some chemical bonding concepts that arise from group-subgroup relationships during phase transitions in these materials. It is clear that these layered chalcogenides support square lattices where electronic instabilities give way to either bonding distortions or superconductivity.
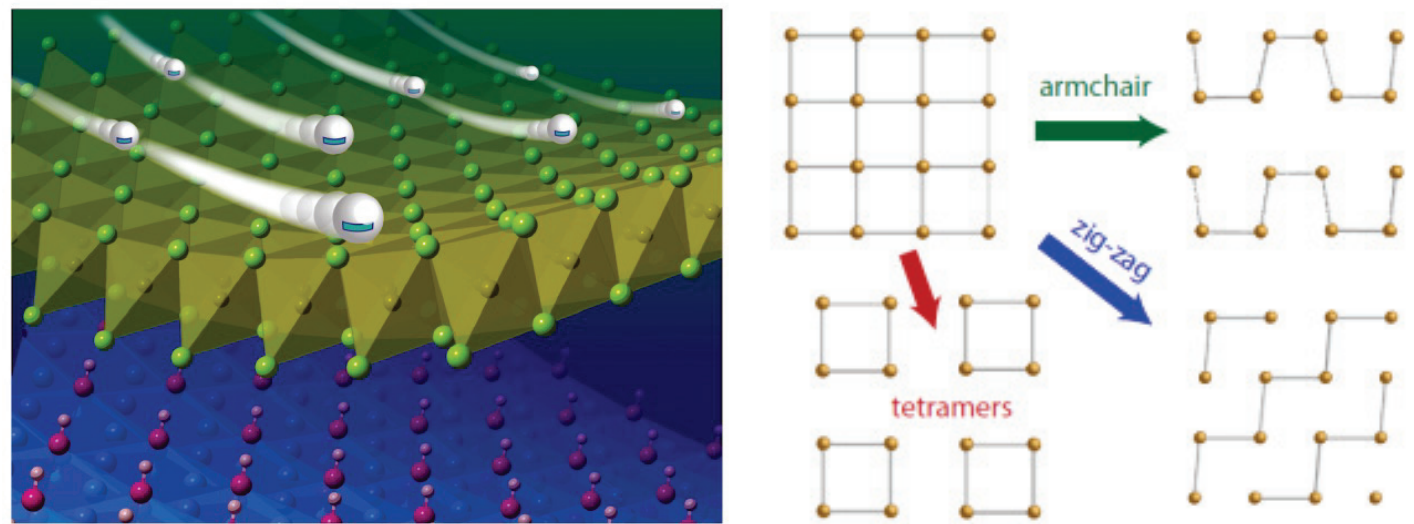

Figures from (left) Zhou et al, J. Mater. Chem. C 2016, 4, 3934 and (right) Zhou and Rodriguez, Chem. Mater. 2017, 29, 5737. 